\section{Eisenmangel oft unentdeckt und unbehandelt}

\author{
Rund $10-30 \%$ der Patienten mit chronisch obstruktiver \\ Lungenerkrankung (COPD) haben eine Anämie. In 2 Teilstudien \\ haben D. S. Silverberg et al. untersucht, wie oft ein Eisenmangel \\ die Ursache ist und ob eine Substitutionstherapie positive \\ Effekte auf die COPD hat. \\ BMC Pulm Med 2014; 14: 24
}

In der 1. Teilstudie wurden mittels Krankenhausdaten die Prävalenz und Behandlung von Anämie und Eisenmangel bei 107 Patienten untersucht, die wegen einer akuten COPD-Exazerbation in ein Krankenhaus aufgenommen worden waren. Im 2. Studienabschnitt wurden 12 ambulante anämische Patienten mit COPD und chronischer Niereninsuffizienz mit einer Kombination aus Erythropoese stimulierenden Agenzien und intravenösem Eisen behandelt. 47 der Patienten (43,9\%) hatten bei Aufnahme einen Hämoglobin
(Hb)-Wert von < $12 \mathrm{~g} / \mathrm{dl}$. Nur in 18 Fällen $(38,3 \%)$ wurde der Eisenstatus bestimmt. Obwohl bei allen 18 Patienten ein Eisenmangel bestätigt wurde, erfolgte bei keiner Person eine Substitutionsbehandlung, weder im Krankenhaus noch nach der Entlassung. Alle 12 anämischen Patienten aus Teilstudie 2 erhielten eine 5-wöchige Substitutionstherapie mit Eiseninfusionen (Gesamtdosis $1000 \mathrm{mg}$ ) und Epoetin beta (wöchentlich 10000 IE s.c.). Eine Woche später wurden die hämatologische Antwort und die Schwere der Dyspnoe er- fasst. Letztere wurde mittels visueller Analogskala (VAS) ermittelt.

11 Patienten hatten einen Eisenmangel. Serumferritin und Transferrinsättigung waren nach der Therapie von 99 auf $330,6 \mathrm{ng} / \mathrm{ml}$ bzw. von 12,8 auf $24,0 \%$ gestiegen (jeweils $p=0,005$ ). Der Hb-Wert stieg von 9,9 auf $12,35 \mathrm{~g} / \mathrm{dl}(\mathrm{p}=0,0005)$, der Hämatokrit (Hkt)-Wert von 29,9 auf $38,1 \%(p=0,0005)$ und die Erythrozytenzahl von 3,3 auf 3,9×106/ $\mu \mathrm{l}(\mathrm{p}=0,01)$. Der VAS-Score stieg von 2,5 auf 8,5 ( $\mathrm{p}=0,0005)$. Diese Verbesserung stand in direktem $\mathrm{Zu}$ sammenhang mit dem Anstieg von Hbund Hkt-Wert.

\section{Fazit}

Die Prävalenz von Anämie und Eisenmangel bei COPD war laut den Autoren sehr hoch. Dennoch werde selten nach Eisenmangel gesucht oder dieser behandelt. Seine Behebung mittels Erythropoese stimulierender Agenzien und intravenösem Eisen könnte auch die Dyspnoe und damit die Lebensqualität verbessern.

Dr. Susanne Krome, Melle

ten $76 \%$ eine Thromboseprophylaxe erhalten. Unabhängige Risikofaktoren waren Adipositas, Hypertonie und kardiovaskuläre Erkrankungen. Bei 39\% trat die LE im Krankenhaus auf. Die Diagnose wurde im Mittel an Tag 23 nach Trauma/Operation gestellt. Die Mortalitätsrate nach LE lag bei 0,07\% im Gesamtkollektiv und bei $15,29 \%$ in der Kohorte. Eine tiefe Venenthrombose als Emboliefokus konnte nur bei einem Drittel aller Patienten gesichert werden. Hatte ein Patient 2 oder mehr Begleiterkrankungen, war seine Mortalität um das 3,5-fache erhöht. Zwischen Ausmaß des initialen Traumas und LE-Risiko bestand kein statistisch signifikanter $\mathrm{Zu}$ sammenhang.

\section{Fazit}

Bei orthopädischen und Traumapatienten ist die Lungenembolie-Rate deutlich erhöht, die Mortalität liegt jedoch niedriger als in älteren Daten ermittelt. Internistische Risikofaktoren erhöhen das Risiko mehr als das Ausmaß eines Traumas, so die Autoren.

\section{Dr. Christoph Feldmann, Köln} bolien (LE) können zu den venösen thromErkrankungen gezäh Gesamtbevölkerung auf. Etwa 5-10\% aller Todesfälle im Krankenhaus sind auf eine LE zurückzuführen. Bei chirurgischen Patienten ist sogar jeder 2 . betroffen, wenn keine Thromboseprophylaxe durchgeführt wird. Genauere Daten für das Auftreten einer LE bei orthopädischen und Traumapatienten wurden nun erhoben. Zentrum der Kohortenstudie war ein gro- ßes Lehrkrankenhaus in England. Alle Paakuten Traumas oder einer geplanten orthopädischen Operation aufgenommen geschlossen und für 6 Monate verfolgt Patienten mit den klinischen Zeichen einer LE erhielten eine Angio-Computertomografie, um eine Embolie zu sichern oder auszuschließen.

Bei $85(0,47 \%)$ von 18151 Patienten trat in diesem Zeitraum eine LE auf. Davon hat- 